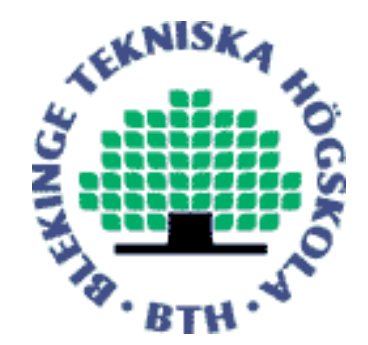

Copyright @ 2013 IEEE.

Citation for the published paper:

Title:

Fast Time-Domain Algorithms for UWB Bistatic SAR Processing

Author:

Viet Thuy Vu, Thomas Sjögren, Mats Pettersson

Journal:

IEEE Transactions on Aerospace and Electronic Systems

Year:

2013

Vol:

49

Issue:

3

Pagination:

1982-1994

URL/DOI to the paper:

10.1109/TAES.2013.6558032

This material is posted here with permission of the IEEE. Such permission of the IEEE does not in any way imply IEEE endorsement of any of BTH's products or services Internal or personal use of this material is permitted. However, permission to reprint/republish this material for advertising or promotional purposes or for creating new collective works for resale or redistribution must be obtained from the IEEE by sending a blank email message to pubs-permissions@iee.org.

By choosing to view this document, you agree to all provisions of the copyright laws protecting it. 


\section{Fast Time-Domain Algorithms for UWB Bistatic SAR Processing}

VIET T. VU, Member, IEEE

THOMAS K. SJÖGREN, Student Member, IEEE

MATS I. PETTERSSON, Member, IEEE

Blekinge Institute of Technology

Sweden

Two fast time-domain algorithms are introduced for ultrawideband-ultrawidebeam (UWB) bistatic synthetic aperture radar (SAR) processing; they are bistatic fast backprojection (BiFBP) and bistatic fast factorized backprojection (BiFFBP). Both algorithms process radar echoes on a subaperture and subimage basis in order to minimize processing time. They are shown to work with any configuration of bistatic SAR. They also own time-domain characteristics, which are essential for UWB radar signal processing. BiFBP and BiFFBP are experimented successfully on the CARABAS-II simulated data.

Manuscript received February 20, 2012; revised June 20 and November 1, 2012; released for publication January 16, 2013.

IEEE Log No. T-AES/49/3/944625.

Refereeing of this publication was handled by F. Gini.

This research project was supported by the KK-Foundation.

Authors' address: Department of Electrical Engineering, Blekinge Institute of Technology, Campus Gräsvik, Karlskrona 37143, Sweden, E-mail: (viet.thuy.vu@bth.se).

0018-9251/13/\$26.00 (c) 2013 IEEE

\section{INTRODUCTION}

Synthetic aperture radar (SAR) plays an important role in geoscience and remote sensing applications. The ability to effectively operate in severe weather conditions is a major advantage of SAR in comparison with other sensor systems.

SAR systems can be classified into ultrawideband-ultrawidebeam (UWB) and narrowband-narrowbeam (NB) groups. The concept of UWB in SAR is clarified here in order to distinguish it from the one of conventional or NB. Hence, UWB SAR refers to a SAR system utilizing large fractional signal bandwidth and wide integration angle associated with wide antenna beamwidth. According to the definition of an ultrawideband signal issued by the FCC in 2002 [1], signals with fractional bandwidth larger than 0.2 or signals with absolute bandwidth larger than $500 \mathrm{MHz}$ are both considered as UWB signals. The experimental SAR systems such as CARABAS-II [2] operating in the lower VHF-band (from 20 to $90 \mathrm{MHz}$ ) and LORA in both VHF- and UHF-bands (from 200 to $800 \mathrm{MHz}$ ) [3] are considered to be UWB SAR systems. The fractional bandwidth of the former is up to 1.17 (much larger than 0.2), while the absolute bandwidth of the latter is nominated to be $600 \mathrm{MHz}$. Both systems utilize dipole antennas, and the integration angle associated with the antenna beamwidth is up to $110^{\circ}$. As the resolution of a SAR system depends strongly on the radar signal bandwidth and the antenna beamwidth [4], high resolutions in azimuth (along track) and range (cross track) can be achieved simultaneously only with UWB SAR systems. For this reason UWB SAR is mostly used in high-resolution ground imaging. There are also other important applications of UWB SAR such as change detection in dense forested areas or under camouflage [5] and ground moving target detection and estimation [6,7], which are of interest to both military and civilian end-users. However, UWB SAR signal processing is very challenging, which has been partially indicated in [8]. UWB SAR systems' associated long integration time usually generates a huge amount of data, large range migration (RM), and big motion error. For example, the dimensions of the CARABAS-II data matrix can be up to $35840 \times 8192$. Processing such data cannot rely on the frequency-domain algorithms such as range Doppler (RD) [9], RM [10], and chirp scaling (CS) [11]. Unlike NB SAR the azimuth and range coupling is serious for UWB SAR, and most approximations for NB SAR are therefore not totally valid for UWB SAR [12]. As the frequency-domain algorithms work with whole SAR data matrices instead of a single SAR data vector, the coupling needs to be taken into account by these algorithms [13]. Also, since they work with the whole SAR data matrix, these algorithms do not support real-time processing. As shown in [14] 
signal processing for UWB SAR usually relies on the time-domain algorithms like global backprojection (GBP) [15].

SAR systems can also be categorized into monostatic and bistatic groups depending on the relative positions of transmitter and receiver. Monostatic SAR is interpreted as SAR systems utilizing colocated transmitter and receiver, while bistatic SAR refers to SAR systems whose transmitter and receiver(s) are separated. Some bistatic SAR experiments have been performed recently using the existing monostatic SAR systems. For example, the bistatic experiments with TerraSAR-X satellite [16] and the airborne SAR-sensor PAMIR [17] are given in [18]. Another example can be found from the bistatic field campaign performed by LORA [3] and SETHI [19] which are presented in [20]. In general such bistatic SAR systems have a better ability to avoid jammer in comparison with monostatic SAR systems illuminating the same ground scene. Processing SAR data normally requires much effort and sometimes may only be performed at ground stations. This can be easily handled with the flexibility in deploying receiver(s) of bistatic SAR systems. With the multiple deployed receivers, an object on a ground scene can be observed at different angles with a bistatic SAR system. This enhances the classification ability of that object. From a system designer's point of view, designing bistatic SAR is more flexible than that of monostatic SAR, and the cost to build a bistatic system may therefore be minimized.

Due to unique advantages of bistatic SAR, there has been much research on this topic. Modifying the currently used monostatic SAR algorithms to process bistatic SAR data is focused on which frequency-domain algorithms RD, RM, and CS receive a great amount of interest. In [21] a discussion on bistatic SAR processing using RD is presented. The modified algorithm is stated to work with the azimuth-invariant bistatic configuration where the flight tracks of the transmitter and receiver platforms are parallel and the velocities of these platforms must be identical. Such requirements are not always satisfied in reality. In [22] an approximated RM type processor for the bistatic SAR has been developed. Similar to RD for bistatic, this algorithm is also limited by the spatial-invariant configuration. One of the most successful attempts is the research work in [23]. The nonlinear CS is adapted to handle a general case of bistatic data. As declared this algorithm can handle data with more complicated bistatic geometries than the previously proposed algorithms. Using monostatic algorithms such as RD and CS for bistatic SAR are also proposed in [24] recently. The methods are based on a reference spectrum which is derived by using the two-dimensional (2D) principle of stationary phase. Among the currently used time-domain algorithms for monostatic SAR, only GBP [15] can be applied directly to bistatic cases. Using GBP to reconstruct a SAR scene in a ground plane for bistatic cases, so-called bistatic GBP (BiGBP), has been presented in [25]. A similar investigation, known as a beamforming algorithm, can also be found in [26]. Other time-domain algorithms for monostatic SAR require modifications to be used for bistatic cases. Fast factorized backprojection (FFBP) has been investigated to extend to bistatic [20]. This extended version has been tested with the bistatic SAR system built on the monostactic systems LORA and SETHI. In another investigation FFBP is applied for a geometry of a spaceborne-airborne bistatic SAR configuration [27]. In this special case the elliptical range coordinate and the angular coordinate are only referenced to the transmitter trajectory. A more general bistatic method is introduced in [28], where the reconstruction of SAR scenes is recommended in a ground plane instead of in a range plane and the elliptical range coordinate and the angular coordinate necessarily depend on both transmitter trajectory and receiver trajectory. However, the bistatic algorithms introduced in this paper work with a subaperture and subimage basis and do not require polar grids.

A combination of UWB and bistatic in SAR is expected to bring significant advantages. However, this combination may also bring more challenges in signal processing for UWB bistatic SAR. The objective of this paper is to introduce fast time-domain algorithms to process UWB bistatic SAR data. They are named bistatic fast backprojection (BiFBP) and bistatic fast factorized backprojection (BiFFBP). Please note that BiFFBP presented in this study is different from the one introduced in [20] as the beam center lines of these algorithms are defined differently. Also, the objective of this study is to derive BiFBP and BiFFBP analytically, whereas the experimental results have been focused in [20]. The algorithms presented here inherit the time-domain characteristics such as unlimited scene size, real-time processing, local processing, manageable motion compensation, and large RM handling and are proved to be not limited by any bistatic SAR configuration. The algorithms should work with any platform speed, any flight track, and any flight altitude. The processing inside the algorithms is performed on a subaperture and subimage basis. Such a method of processing allows us to reduce the computational load significantly. The presented algorithms are tested on the different simulated UWB bistatic SAR data corresponding to the different bistatic configurations. A part of this work has been published in [25], [29].

The paper is organized as follows. Section II reviews the GBP algorithm and the applicability of this algorithm to bistatic UWB SAR. BiFBP and BiFFBP are introduced in Section III, and Section IV, 


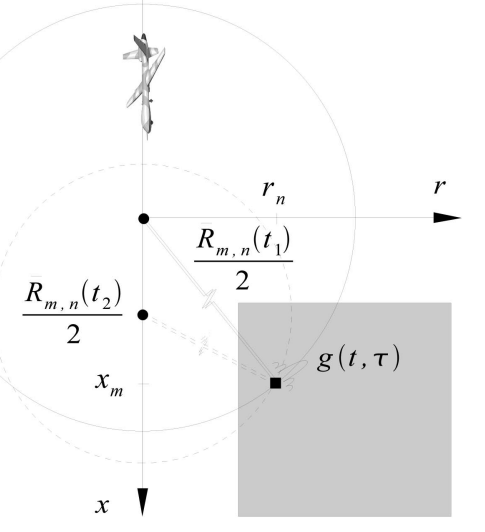

Fig. 1. Illustration of GBP for mono-static SAR. Backprojection of radar echoes into range plane $(x, r)$.

respectively. Experimental results and evaluations are given in Section IV. Section V provides the conclusions.

\section{GBP FOR MONO- AND BISTATIC UWB SAR}

The GBP algorithm [15] is interpreted as a linear and direct transformation process from radar echoes into a complex SAR image. GBP was developed in the 1980s and recognized to be one among SAR algorithms offering referenced SAR images. For monostatic SAR, if the range-compressed radar signal is given by $\bar{g}(t, \tau)$, where $t$ and $\tau$ indicate azimuth-time (slow-time) and range time (fast-time), respectively, the superposition of backprojected radar echoes to reconstruct the imaged scene in the range plane $\left(x_{m}, r_{n}\right)$ is mathematically represented by the integral

$$
h\left(x_{m}, r_{n}\right)=\int_{-t_{i} / 2}^{+t_{i} / 2} \bar{g}\left(t, \frac{\bar{R}_{m, n}(t)}{\mathrm{c}}\right) d t
$$

where $t_{i}$ is the integration time and $\mathrm{c}$ is the speed of light. The two-way traveling distance of a radar pulse $R$ is calculated by

$$
\bar{R}_{m, n}(t)=2 \sqrt{\left(v_{p l} t-x_{m}\right)^{2}+r_{n}^{2}}
$$

where $v_{p l}$ is the speed of the platform. Figure 1 illustrates the backprojection of the radar echoes into the range plane $(x, r)$.

For monostatic GBP the backprojection of radar echoes into a range plane is performed over a spherical mapping. The center of the sphere is determined by the actual aperture position of the platform and the radius is given by the range $\bar{R}_{m, n}(t) / 2$. The image sample $\left(x_{m}, r_{n}\right)$ and other samples belonging to the reconstructed SAR scene, which have the same range $\bar{R}_{m, n}(t) / 2$ given by (2), i.e., crossed by the solid circle in Fig. 1, are assigned the same sample

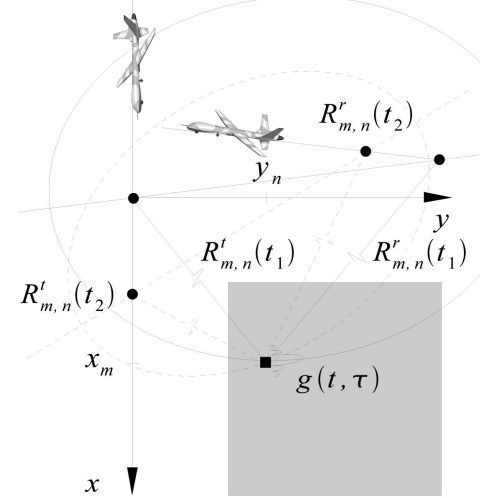

Fig. 2. Illustration of GBP for bistatic SAR. Backprojection of radar echoes into ground plane $(x, y)$.

of the range-compressed radar echoes matched to this range.

Since the two-way traveling distance of a radar pulse is calculated for each aperture position and each image sample in the SAR scene reconstruction of GBP, applying GBP to bistatic is natural. For bistatic cases there is no exact range plane as the plane formed by the flight track of the transmitter platform and the radar pulse incident vectors is not the same as the one formed by the flight track of the receiver platform and the vectors of radar pulse reflection. The reconstructed SAR scene is therefore recommended to be in the ground plane instead of the range plane to simplify the calculation of the traveling distance of a radar pulse. Hence, the mathematic expression of the BiGBP is given by

$$
h\left(x_{m}, y_{n}\right)=\int_{-t_{i} / 2}^{+t_{i} / 2} g\left(t, \frac{R_{m, n}(t)}{\mathrm{c}}\right) d t .
$$

Please note that the range-compressed radar echo $g(t, \tau)$ in (3) is not the same as $\bar{g}(t, \tau)$ due to the difference in geometry. Hence, the traveling distance of a radar pulse $R_{m, n}(t)$ in (3) depends strongly on the movements of the platforms and is now calculated by

$$
\begin{aligned}
R_{m, n}(t)= & R_{m, n}^{t}(t)+R_{m, n}^{r}(t) \\
= & \sqrt{\left(v_{x}^{t} t-x_{m}\right)^{2}+\left(v_{y}^{t} t-y_{n}\right)^{2}+\left(h^{t}\right)^{2}} \\
& +\sqrt{\left(v_{x}^{r} t-x_{m}\right)^{2}+\left(v_{y}^{r} t-y_{n}\right)^{2}+\left(h^{r}\right)^{2}}
\end{aligned}
$$

where the upper subscripts $t$ and $r$ denote the transmitter and receiver sides, while the lower subscripts $x$ and $y$ refer to the components in those directions. Hence, $v_{x}^{t}$ indicates the speed component in the $x$-direction of the transmitter platform and so forth. The flight altitudes of the platforms are denoted by $h^{t}$ and $h^{r}$. The backprojection of the range-compressed radar signal into a ground plane $(x, y)$ is illustrated in Fig. 2. An ellipsoidal mapping is the basic for this backprojection. The foci of the 
ellipsoid are defined by the actual aperture positions of the transmitter and receiver platforms. These foci also determine the major axis of the ellipsoid. The traveling distance of a radar pulse $R$ is nothing else but the major axis of the ellipsoid. The image sample $\left(x_{m}, y_{n}\right)$ and other samples belonging to the reconstructed SAR scene, which match to the distance $R_{m, n}(t)$ given by (4), i.e., crossed by the solid ellipse in Fig. 2, are assigned the same sample of the range-compressed radar echoes corresponding to this distance.

In BiGBP the calculation of $R_{m, n}(t)$ is applied for all aperture positions and all SAR image samples and thus requires a high computational burden. The processing time of BiGBP is clearly proportional to the numbers of aperture positions $N_{l}$, image azimuth samples $N_{x}$, and image ground range samples $N_{y}$. If $\Xi_{\mathrm{BiGBP}}$ is the number of operations required by BiGBP, this number is given by

$$
\Xi_{\text {BiGBP }} \propto N_{l}\left(N_{x} \times N_{y}\right) .
$$

This computational burden is exchanged by a series of unique advantages of BiGBP. For example, BiGBP supports real-time processing. This means that a radar echo impinging on the receiver of the bistatic system can be processed directly without delay. Processing one by one vector of radar signals instead of a matrix of radar signals does not require a large memory and a powerful computer. It is therefore not necessary to process SAR data at ground stations with BiGBP. This further increases the flexibility in designing bistatic SAR systems. The traveling distance calculation based on each aperture position and each image sample in BiGBP also means that the motion errors are compensated automatically in BiGBP. The scene size illuminated by a bistatic SAR system can be seen to be unlimited by the algorithm and depends strongly on the antenna beamwidths, the integration time, the flight attitudes, the radiated power, and the pulse repetition frequency (PRF) of that system. Handling extremely large RM is definitely not the trouble of the algorithm. The reconstructed SAR scene $\left(N_{x} \times N_{y}\right)$ can be selected as desired. On account of the local processing characteristic, BiGBP is quite suitable for processing specified areas of interest. The calculations in BiGBP to reconstruct an illuminated SAR scene are independent of bistatic configuration. This means that the speeds, the flight tracks, and the flight altitudes of transmitter and receiver platforms do not affect the operation of this algorithm. For these reasons GBP can be considered as one of the candidates for UWB bistatic SAR data processing.

\section{FAST BACKPROJECTION FOR UWB BISTATIC SAR}

As discussed in the previous section, beside the unique advantages of BiGBP, the major shortcoming of BiGBP is the high computational burden required by this algorithm. A fast time-domain algorithm, named BiFBP, is introduced in this section. Similar to BiGBP the illuminated scene is supposed to be reconstructed in the ground plane instead of the range plane to simplify the range calculations.

BiFBP processes SAR data on a subaperture and subimage basis, i.e., local processing [30]. Thus, the full reconstructed SAR scene is segmented into $K$ subimages, while the complete transmitter and receiver apertures are split into $L$ subapertures. The reconstruction of the imaged scene is processed in two stages: beamforming and backprojection.

In the beamforming stage all radar echoes $g(t, \tau)$ belonging to the $l$ th subapertures are first shifted in range time $\tau$ (fast-time) with respect to the center of the $k$ th subimage. The shifting in range time means that the origin of the range time axis $\tau$ in processing is not $\tau=0$ but instead $\tau=R_{l, k}(t) / \mathrm{c}$, where $R_{l, k}(t)$ is the traveling distance of a radar pulse radiated from an aperture position belonging to the $l$ th transmitter subaperture impinging on the center of the $k$ th subimage and then reflected to the corresponding aperture position belong to the $l$ th receiver subaperture. The distance $R_{l, k}(t)$ is estimated by

$$
\begin{aligned}
R_{l, k}(t)= & \sqrt{\left(x_{l}^{t}(t)-x_{k}\right)^{2}+\left(y_{l}^{t}(t)-y_{k}\right)^{2}+\left(h^{t}\right)^{2}} \\
& +\sqrt{\left(x_{l}^{r}(t)-x_{k}\right)^{2}+\left(y_{l}^{r}(t)-y_{k}\right)^{2}+\left(h^{r}\right)^{2}}
\end{aligned}
$$

where $\left(x_{k}, y_{k}\right)$ are the coordinates of the center of the $k$ th subimage in the ground plane. Aperture positions belonging to the $l$ th subapertures are denoted by $\left(x_{l}^{t}(t), y_{l}^{t}(t)\right)$ and $\left(x_{l}^{r}(t), y_{l}^{r}(t)\right)$. The shifted radar echo in range time $\tau$ is now given by $g\left(t, \tau+R_{l, k}(t) / \mathrm{c}\right)$. A superposition of these shifted radar echoes then results in the beam $b_{l, k}(\tau)$. The superposition can be interpreted as the projection of the data samples into the beam samples over an elliptical mapping and is illustrated in Fig. 3. If $t_{l}$ is the time instant corresponding to the $l$ th centers of the subapertures and $t_{s}$ the integration time, the beamforming procedure will mathematically be represented by the integral

$$
b_{l, k}(\tau)=\int_{t_{l}-t_{s} / 2}^{t_{l}+t_{s} / 2} g\left(t, \tau+\frac{R_{l, k}(t)}{\mathrm{c}}\right) d t .
$$

The number of beam samples $b_{l, k}(\tau)$ is selected to be just enough to cover the Nyquist sampling subimage. As shown in Fig. 3, a minimum selection of the beam length is limited by two solid-line ellipses.

In the next stage the image samples belonging to the $k$ th subimage are then backprojected by the samples of the $k$ th beam. The backprojection is also performed over an ellipsoidal mapping in which the the foci of the ellipsoid are defined by the $l$ th centers of the transmitter and receiver subapertures. If the subaperture and subimage are small enough, 


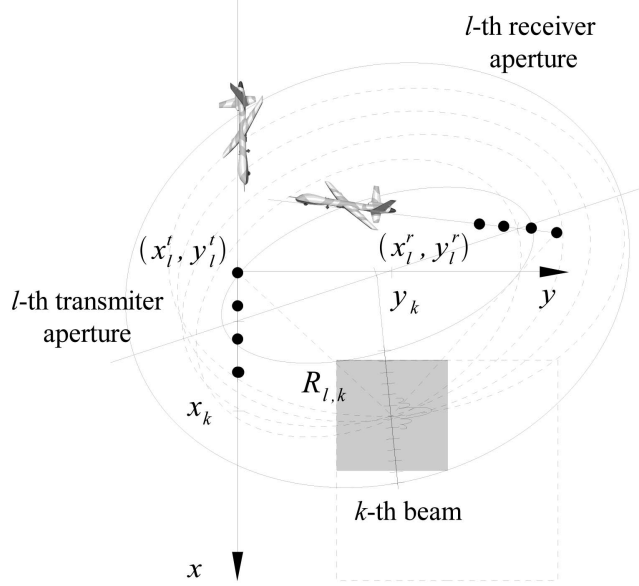

Fig. 3. Beamforming in BiFBP. Beamforming is superposition of radar echo belonging to one transmitter subaperture and one receiver subaperture and illustrated by dashed-line ellipsoids. Solid-line ellipsoids limit beam length.

the ellipsoid mapping can be approximated to a linear mapping and the computational complexity can therefore be reduced. A line going through two foci is nothing else but the the major axis of the ellipsoid. The backprojecting the $i$ th sample of the $k$ th beam to the image sample $\left(x_{m, k}, y_{n, k}\right)$ belonging to the $k$ th subimage is mathematically expressed by

$$
h_{k}\left(x_{m, k}, y_{n, k}\right)=\sum_{l=1}^{L} b_{l, k}\left(\frac{R_{m, n, l, k}^{c}-R_{l, k}^{c}}{\mathrm{c}}\right)
$$

where

$$
\begin{aligned}
R_{m, n, l, k}^{c}= & \sqrt{\left(x_{l}^{t, c}-x_{m, k}\right)^{2}+\left(y_{l}^{t, c}-y_{n, k}\right)^{2}+\left(h^{t}\right)^{2}} \\
& +\sqrt{\left(x_{l}^{r, c}-x_{m, k}\right)^{2}+\left(y_{l}^{r, c}-y_{n, k}\right)^{2}+\left(h^{r}\right)^{2}}
\end{aligned}
$$

and

$$
\begin{aligned}
R_{l, k}^{c}= & \sqrt{\left(x_{l}^{t, c}-x_{k}\right)^{2}+\left(y_{l}^{t, c}-y_{k}\right)^{2}+\left(h^{t}\right)^{2}} \\
& +\sqrt{\left(x_{l}^{r, c}-x_{k}\right)^{2}+\left(y_{l}^{r, c}-y_{k}\right)^{2}+\left(h^{r}\right)^{2}} .
\end{aligned}
$$

The upper subscript $c$ in (9) and (10) refers to the centers of the subapertures. Thus, $\left(x_{l}^{t, c}, y_{l}^{t, c}\right)$ and $\left(x_{l}^{r, c}, y_{l}^{r, c}\right)$ are the coordinates of the centers of the $l$ th subapertures. As illustrated in Fig. 4, the distance $R_{m, n}^{c}$ defines the major axis of an ellipse of this backprojection. Over an ellipsoidal mapping other image samples belonging to the $k$ th subimage having the same value of $R_{m, n, l, k}^{c}$ with respect to the centers of the $l$ th subapetures, or in other words crossed by this ellipse, are also assigned by this $i$ th sample. $R_{l, k}^{c}$ refers to the $k$ th beam center and also indicates the major axis of another ellipse crossing the center of the $k$ th subimage. This procedure is repeated for all subapertures. The sampled version of the $k$ th

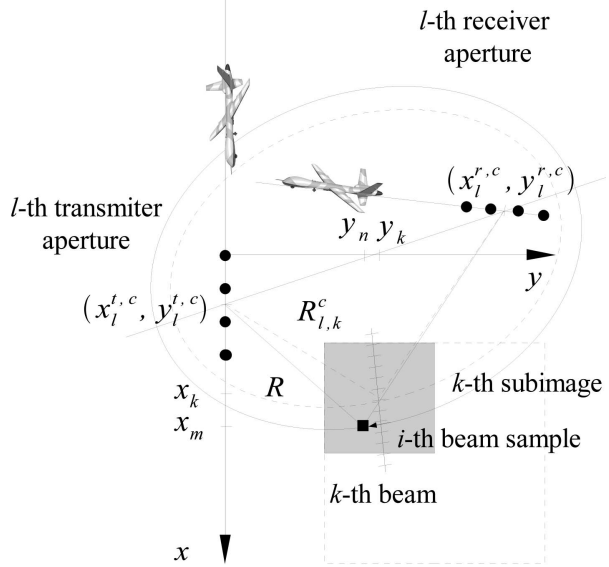

Fig. 4. Local backprojection in BiFBP. Backprojection is performed over elliptical mapping. Centers of $l$ th subapertures define elements of mapping ellipsoids. Mapping from formed beam to arbitrary image sample is illustrated by solid-line ellipsoid.

subimage can be presented in the matrix form as follows

$$
\mathbf{H}_{k}=\left(\begin{array}{ccc}
h_{k}\left(x_{1, k}, y_{1, k}\right) & h_{k}\left(x_{2, k}, y_{1, k}\right) & \ldots \\
h_{k}\left(x_{1, k}, y_{2, k}\right) & \ddots & \vdots \\
\vdots & \ldots & \ddots
\end{array}\right) .
$$

The full SAR image is finally retrieved by a union of all subimages in a correct order. The union operation depends on how the full SAR image is segmented in the beginning. Let us consider a simple example where a square full SAR image is equally segmented by an integer factor of $\sqrt{K}$ in the $x$-direction and also by the same factor in the $y$-direction. In this case the sampled version of the full SAR image is given by

$$
\mathbf{H}=\left(\begin{array}{cccc}
\mathbf{H}_{1} & \mathbf{H}_{2} & \cdots & \mathbf{H}_{\sqrt{K}} \\
\mathbf{H}_{1+\sqrt{K}} & \mathbf{H}_{2+\sqrt{K}} & \cdots & \mathbf{H}_{2 \sqrt{K}} \\
\vdots & \ddots & \ddots & \vdots \\
\mathbf{H}_{1+(\sqrt{K}-1) \sqrt{K}} & \mathbf{H}_{2+(\sqrt{K}-1) \sqrt{K}} & \cdots & \mathbf{H}_{K}
\end{array}\right) .
$$

If we use $U$ to denote the union of all subimages in a correct order, the final expression of the BiFBP algorithm can be formulated by

$$
\begin{aligned}
& \bigcup_{k=1}^{K} h_{k}\left(x_{m, k}, y_{n, k}\right) \\
& \quad=\bigcup_{k=1}^{K} \sum_{l=1}^{L} \int_{t_{l}-t_{s} / 2}^{t_{l}+t_{s} / 2} g\left(t, \frac{R_{m, n, l, k}^{c}-R_{l, k}^{c}+R_{l, k}(t)}{\mathrm{c}}\right) d t .
\end{aligned}
$$

If we compare (3) and (13), we see that BiFBP is only an approximation version of BiGBP. Hence, $R_{m, n}(t)$ in (3) is approximated by $R_{m, n, l, k}^{c}$ in (13), 
whereas also in (13), $R_{l, k}^{c}$ is approximated by $R_{l, k}(t)$. To be sure that these approximations are valid, subaperture length and subimage area in BiFBP must be selected so that the far field condition is fulfilled. A combination of the approximations results in $R_{m, n, l, k}^{c}-R_{l, k}^{c}+R_{l, k}(t) \approx R_{m, n}(t)$.

Unlike BiGBP, whose processing time is proportional to the number of aperture positions $N_{l}$ and the reconstructed SAR scene $\left(N_{x} \times N_{y}\right)$, the computational cost of BiFBP depends also on the selections of subaperture length and subimage area. If the algorithm processes the radar echoes on a basic of $L$ subapertures and $K$ subimages, the needed number of operations for each pair of subapertures and each subimage is proportional to

1) forming one beam with $N_{b}$ samples: $\left(N_{l} N_{b}\right) / L$.

2) backprojecting one beam to one subimage: $\left(N_{x} \times N_{y}\right) / K$.

For all subapertures and subimages, the number of operations are counted by

$$
\Xi_{\mathrm{BiFBP}} \propto L K\left[\frac{N_{l} N_{b}}{L}+\frac{\left(N_{x} \times N_{y}\right)}{K}\right] .
$$

In reality the number of aperture positions of the transmitter and receiver subapertures or the subaperture length $\left(N_{l} / L\right)$ is inversely proportional to the subimage size, i.e., the square root of the square subimage area

$$
\frac{N_{l}}{L}=\gamma \sqrt{\frac{K}{\left(N_{x} \times N_{y}\right)}}
$$

where $\gamma$ is an integer number and $\gamma \gg 1$. Equation (14) can therefore be rewritten by

$$
\Xi_{\mathrm{BiFBP}} \propto N_{l}\left[N_{b} K+\frac{1}{\gamma} \sqrt{\frac{\left(N_{x} \times N_{y}\right)^{3}}{K}}\right] .
$$

The first derivative of a function is equal to zero at minimum. Thus, to find the minimum of (16), we need to find the $\operatorname{root}(\mathrm{s}) K$ that the first derivative of $\Xi_{\mathrm{BiFBP}}$ with respect to $K$ is zero.

$$
\begin{aligned}
\frac{\partial \Xi_{\text {BiFBP }}}{\partial K} & =0 \\
N_{l}\left[N_{b}-\frac{1}{2 \gamma} \sqrt{\frac{\left(N_{x} \times N_{y}\right)^{3}}{K^{3}}}\right] & =0 \\
K & =\frac{N_{x} \times N_{y}}{\sqrt[3]{4 \gamma^{2} N_{b}^{2}}} .
\end{aligned}
$$

Substituting the found value of $K$ in (17) to (16) results in the optimum number of operations required by BiFBP as

$$
\Xi_{\mathrm{BiFBP}} \propto N_{l}\left(N_{x} \times N_{y}\right) \sqrt[3]{\frac{6.75 N_{b}}{\gamma^{2}}} .
$$

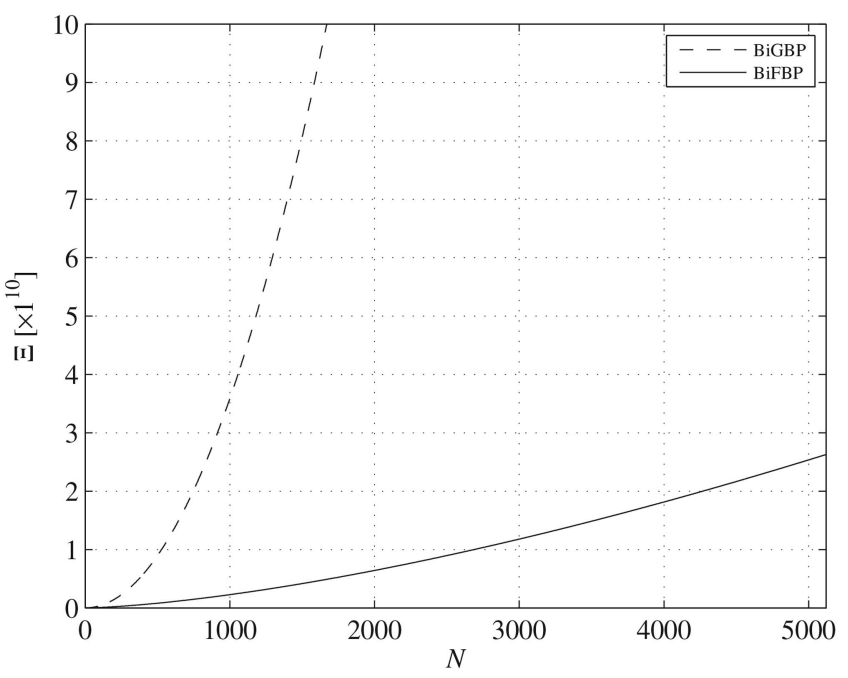

Fig. 5. Number of operations required by BiGBP and BiFBP for case $N_{x}=N_{y}=N_{l}=N$ and $L=\sqrt{K}=\sqrt{N}$.

A comparison between (5) and (18) shows clearly that BiFBP has advantages of processing time over BiGBP. The cube root term in (18) decides how many times BiFBP runs faster than BiGBP. Let us take a simple example to examine the processing time of BiFBP in comparision with BiGBP. If we assume that the reconstructed SAR scene is square, i.e., $N_{x} \times N_{y}=N^{2}$ samples and $N$ is also assumed to be the number of aperture positions, i.e., $N_{l}=N$, the processing time of BiGBP will be proportional to $N^{3}$. For BiFBP we select $L=\sqrt{K}=N_{b}=\sqrt{N}$. Equation (15) results in $\gamma=N$. In this case the number of operations required by BiFBP is proportional to $N^{2} \sqrt{N}$ and therefore less than BiGBP approximately $\sqrt{N}$ times. In other words the processing time of BiFBP is reduced approximately $\sqrt{N}$ times in comparison with that of BiGBP. Figure 5 plots the functions (5) and (18) with different values of $N \in[0,5120]$.

As stated in [31] processing with the subaperture and subimage basis causes range errors in both beamforming and backprojection stages and therefore phase error in the reconstructed SAR scene. The larger the subaperture and the subimage are, the shorter processing time BiFBP requires, however, the bigger phase error BiFBP generates. This trade-off is represented by [31]

$$
\Delta \phi_{\max }=\frac{\pi d_{k}}{4 \lambda_{c} \cos \alpha}\left(\frac{d_{t}}{R_{t, 0}}+\frac{d_{r}}{R_{r, 0}}\right)
$$

where $\lambda_{c}$ denotes the wavelength of the radar signal. The maximum dimension of a subimage is denoted by $d_{k}$. For a square subimage $d_{k}$ is the diagonal of the subimage. The angle $\alpha$ is the maximum bistatic backprojection angle. Transmitter and receiver subaperture lengths are given by $d_{t}$ and $d_{r}$, respectively. The ranges $R_{t, 0}$ and $R_{r, 0}$ are minimum values of $R_{t}$ and $R_{r}$, i.e., minimum ranges. 


\section{FFBP FOR UWB BISTATIC SAR}

BiFBP described in the previous section have been proved to run faster than BiGBP due to processing of BiFBP on a subaperture and subimage basis. However, there exist other methods which can further reduce processing time such as factorization. The factorization in a time-domain algorithm implies multiple stage processing. Another fast time-domain algorithm, called BiFFBP, is presented in this section.

Similar to BiFBP the bistatic SAR data is processed on a subaperture and subimage basis in BiFFBP. However, instead of processing the radar echoes with a single beamforming stage, they are processed with different beamforming stages before the backprojection stage. Hence, in the first beamforming stage, the same procedure for BiFBP is handled. The full reconstructed SAR scene and the complete transmitter and receiver apertures are split into $K_{1}$ first-order subimages and $L_{1}$ first-order subapertures. To form the $k_{1}$ th first-order beam, the bistatic SAR data belonging to the $l_{1}$ th subaperture are first shifted with respect to the center of the $k_{1}$ th subimage then superposed

$$
b_{l_{1}, k_{1}}(\tau)=\int_{t_{l_{1}}-t_{s} / 2}^{t_{l_{1}}+t_{s} / 2} g\left(t, \tau+\frac{R_{l_{1}, k_{1}}(t)}{\mathrm{c}}\right) d t
$$

where $t_{l_{1}}$ is the time instant corresponding to the $l_{1}$ th centers of the subapertures, $t_{s}$ is the integration time along the subapertures, and

$$
\begin{aligned}
R_{l_{1}, k_{1}}(t)= & \sqrt{\left(x_{l_{1}}^{t}(t)-x_{k_{1}}\right)^{2}+\left(y_{l_{1}}^{t}(t)-y_{k_{1}}\right)^{2}+\left(h^{t}\right)^{2}} \\
& +\sqrt{\left(x_{l_{1}}^{r}(t)-x_{k_{1}}\right)^{2}+\left(y_{l_{1}}^{r}(t)-y_{k_{1}}\right)^{2}+\left(h^{r}\right)^{2}} .
\end{aligned}
$$

In (21) $\left(x_{l_{1}}^{t}(t), y_{l_{1}}^{t}(t)\right)$ indicates an aperture position of the $l_{1}$ transmitter subaperture and $\left(x_{l_{1}}^{r}(t), y_{l_{1}}^{r}(t)\right)$ the corresponding aperture position of the $l_{1}$ receiver subaperture. The center of the $k_{1}$ subimage is denoted by $\left(x_{k_{1}}, y_{k_{1}}\right)$. After the first beamforming stage, $K_{1} \times L_{1}$ first-order beams are generated covering $K_{1}$ subimages. The number of beam samples must be selected large enough to cover the beams created in the next beamforming stages.

In the second beamforming stage, the first-order beams are used to form second-order beams. A first-order subimage is further segmented into a number of smaller second-order subimages. The number of subimages therefore increases to $K_{2}$. Conversely, a number of first-order transmitter subapertures is combined together to form a larger second-order subaperture. This step is also applied to the same number of first-order receiver supapertures. Thus, the number of subapetures is reduced to $L_{2}$.

For the $k_{2}$ th second-order beamformation, each beam sample of the $k_{2}$ th second-order beam is created

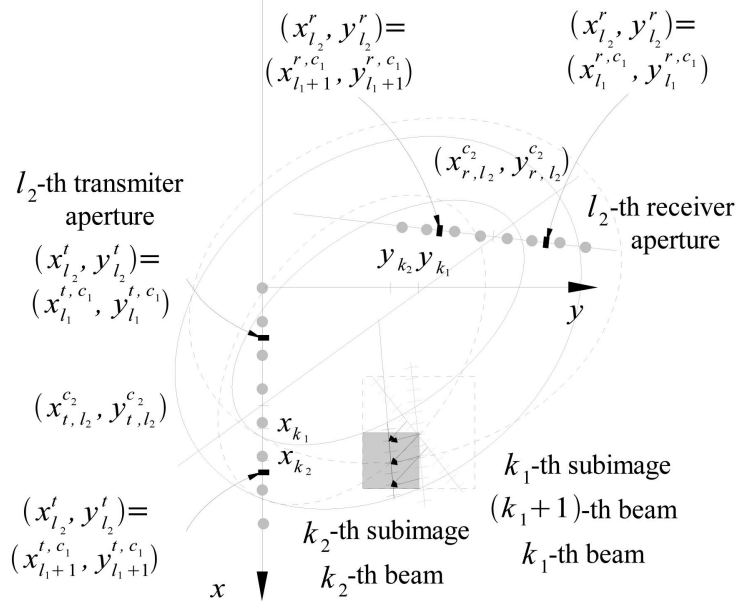

Fig. 6. Second-order beamforming in BiFFBP. $L_{1} / L_{2}$ first-order beams are first shifted and then added together in ellipsoidal mapping to created second-order beam. In this example, $L_{1} / L_{2}=2$ and $K_{2} / K_{1}=4$. Centers of first-order subapertures, e.g. $\left(x_{l_{1}}^{t, c_{1}}, y_{l_{1}}^{t, c_{1}}\right)$, $\left(x_{l_{1}}^{r, c_{1}}, y_{l_{1}}^{r, c_{1}}\right),\left(x_{l_{1}+1}^{t, c_{1}}, y_{l_{1}+1}^{t, c_{1}}\right)$, and $\left(x_{l_{1}+1}^{r, c_{1}}, y_{l_{1}+1}^{r, c_{1}}\right)$, are aperture positions of second-order subaperture, i.e., $\left(x_{l_{2}}^{t}, y_{l_{2}}^{t}\right)$ and $\left(x_{l_{2}}^{r}, y_{l_{2}}^{r}\right)$.

by adding $L_{1} / L_{2}$ beam samples of the first-order beams over an ellipsoidal mapping. The number of beam samples must be selected large enough to cover either the beams created in the next beamforming stage if there are any or the subimages in the backprojection stage. This procedure is illustrated in Fig. 6 and described by

$$
b_{l_{2}, k_{2}}(\tau)=\sum_{l_{1}=1+\left(l_{2}-1\right) L_{1} / L_{2}}^{l_{2} L_{1} / L_{2}} b_{l_{1}, k_{1}}\left(\tau+\frac{R_{l_{1}, k_{2}}^{c_{1}}-R_{l_{1}, k_{1}}^{c_{1}}}{\mathrm{c}}\right)
$$

where

$$
\begin{aligned}
R_{l_{1}, k_{2}}^{c_{1}}= & \sqrt{\left(x_{l_{1}}^{t, c_{1}}-x_{k_{2}}\right)^{2}+\left(y_{l_{1}}^{t, c_{1}}-y_{k_{2}}\right)^{2}+\left(h^{t}\right)^{2}} \\
& +\sqrt{\left(x_{l_{1}}^{r, c_{1}}-x_{k_{2}}\right)^{2}+\left(y_{l_{1}}^{r, c_{1}}-y_{k_{2}}\right)^{2}+\left(h^{r}\right)^{2}}
\end{aligned}
$$

and

$$
\begin{aligned}
R_{l_{1}, k_{1}}^{c_{1}}= & \sqrt{\left(x_{l_{1}}^{t, c_{1}}-x_{k_{1}}\right)^{2}+\left(y_{l_{1}}^{t, c_{1}}-y_{k_{1}}\right)^{2}+\left(h^{t}\right)^{2}} \\
& +\sqrt{\left(x_{l_{1}}^{r, c_{1}}-x_{k_{1}}\right)^{2}+\left(y_{l_{1}}^{r, c_{1}}-y_{k_{1}}\right)^{2}+\left(h^{r}\right)^{2}} .
\end{aligned}
$$

In Fig. 6 the second-order beam is illustrated to be formed by two first-order beams, i.e., $L_{1} / L_{2}=2$, while the second-order subimage is fourth of the first-order subimage, i.e., $K_{2} / K_{1}=4$. Hence, the $k_{2}$ th beam is formed by the first-order $k_{1}$ th and $\left(k_{1}+1\right)$ th beams. After the second beamforming stage, $K_{2} \times L_{2}$ second-order beams are created covering $K_{2}$ subimages. The coordinates of the aperture positions of the second-order subaperture, i.e., $\left(x_{l_{2}}^{t}, y_{l_{2}}^{t}\right)$ and 
$\left(x_{l_{2}}^{r}, y_{l_{2}}^{r}\right)$, are defined by the centers of the first-order subapertures, e.g., $\left(x_{l_{1}}^{t, c_{1}}, y_{l_{1}}^{t, c_{1}}\right),\left(x_{l_{1}}^{r, c_{1}}, y_{l_{1}}^{r, c_{1}}\right),\left(x_{l_{1}+1}^{t, c_{1}}, y_{l_{1}+1}^{t, c_{1}}\right)$, and $\left(x_{l_{1}+1}^{r, c_{1}}, y_{l_{1}+1}^{r, c_{1}}\right)$.

Similarly, in the next beamforming stages, higher order beams are formed from lower order beams. This step is repeated until the final beamforming stage. After each beamforming stage the number of subapertures decreases, i.e., longer subapertures, while the number of subimages increases, i.e., smaller subimages. The number of beam samples is therefore reduced corresponding to the decrease in subimage area.

Consider the example where BiFFBP only has two beamforming stages and the second-order beams are used to backproject into the $K_{2}$ subimages. Similar to BiFBP the backprojection is carried out over an ellipsoidal mapping. The $l_{2}$ th centers of the transmitter and receiver subapertures determine the foci of the ellipse and the major axis of the ellipsoid is the line going through these foci. The backprojecting of the $i_{2}$ th sample of the $k_{2}$ th second-order beam to the image sample $\left(x_{m}, y_{n}\right)$ belonging to the $k_{2}$ th second-order beam is represented by

$$
h_{k_{2}}\left(x_{m, k_{2}}, y_{n, k_{2}}\right)=\sum_{l_{2}=1}^{L_{2}} b_{l_{2}, k_{2}}\left(\frac{R_{m, n, l_{2}, k_{2}}^{c_{2}}-R_{l_{2}, k_{2}}^{c_{2}}}{\mathrm{c}}\right)
$$

where

$$
\begin{aligned}
R_{m, n, l_{2}, k_{2}}^{c_{2}}= & \sqrt{\left(x_{l_{2}}^{t, c_{2}}-x_{m, k_{2}}\right)^{2}+\left(y_{l_{2}}^{t, c_{2}}-y_{n, k_{2}}\right)^{2}+\left(h^{t}\right)^{2}} \\
& +\sqrt{\left(x_{l_{2}}^{r, c_{2}}-x_{m, k_{2}}\right)^{2}+\left(y_{l_{2}}^{r, c_{2}}-y_{n, k_{2}}\right)^{2}+\left(h^{r}\right)^{2}} .
\end{aligned}
$$

and

$$
\begin{aligned}
R_{l_{2}, k_{2}}^{c_{2}}= & \sqrt{\left(x_{l_{2}}^{t, c_{2}}-x_{k_{2}}\right)^{2}+\left(y_{l_{2}}^{t, c_{2}}-y_{k_{2}}\right)^{2}+\left(h^{t}\right)^{2}} \\
& +\sqrt{\left(x_{l_{2}}^{r, c_{2}}-x_{k_{2}}\right)^{2}+\left(y_{l_{2}}^{r, c_{2}}-y_{k_{2}}\right)^{2}+\left(h^{r}\right)^{2}} .
\end{aligned}
$$

In (26) and (27) the coordinates $\left(x_{l_{2}}^{t, c_{2}}, y_{l_{2}}^{t, c_{2}}\right)$ and $\left(x_{l_{2}}^{r, c_{2}}, y_{l_{2}}^{r, c_{2}}\right)$ are the centers of the $l_{2}$ th subapertures. Over an ellipsoid mapping the image samples, which define the same traveling distance $R$ or are crossed by the solid ellipse with the major axis $R$ and belong to the $k_{2}$ th subimage, are mapped by the $i_{2}$ th sample of the $k_{2}$ th second-order beam. This procedure is repeated for all $L_{2}$ subapertures. Finally, $K_{2}$ second-order subimages are arranged to build up $K_{1}$ first-order subimages. These $K_{1}$ first-order subimages are again combined to reconstruct the full reconstructed SAR scene. The final mathematical expression of BiFFBP with two beamforming stages is given in (28).
TABLE I

CARABAS-II and LORA Parameters

\begin{tabular}{ccc}
\hline \hline Parameter & CARABAS-II & LORA \\
\hline The highest frequency processed & $82.5 \mathrm{MHz}$ & \\
The lowest frequency processed & $21.9 \mathrm{MHz}$ & \\
Platform speed $v_{p l}$ & $126 \mathrm{~m} / \mathrm{s}$ & $130 \mathrm{~m} / \mathrm{s}$ \\
Number of aperture positions & 20480 & \\
Aperture step & $0.9375 \mathrm{~m}$ & $0.9673 \mathrm{~m}$ \\
Flight altitude & $3700 \mathrm{~m}$ & $2900 \mathrm{~m}$ \\
Minimum range $R_{0}$ & $5900 \mathrm{~m}$ & $3000 \mathrm{~m}$ \\
PRF & $137 \mathrm{~Hz}$ & \\
\hline
\end{tabular}

$$
\begin{aligned}
& \bigcup_{k_{1}=1}^{K_{1}} \bigcup_{k_{2}=1+\left(k_{1}-1\right) K_{2} / K_{1}}^{k_{1} K_{2} / K_{1}} h_{k_{2}}\left(x_{m, k_{2}}, y_{n, k_{2}}\right) \\
& =\bigcup_{k_{1}=1}^{K_{1}} \bigcup_{k_{2}=1+\left(k_{1}-1\right) K_{2} / K_{1}}^{k_{1} K_{2} / K_{1}} \sum_{l_{2}=1}^{L_{2}} \sum_{l_{1}=1+\left(l_{2}-1\right) L_{1} / L_{2}}^{l_{2} L_{1} / L_{2}} \int_{t_{1}-t_{s} / 2}^{t_{1}+t_{s} / 2} \\
& \quad \times g\left(t, \frac{\left(R_{m, n, l_{2}, k_{2}}^{c_{2}}-R_{l_{2}, k_{2}}^{c_{2}}\right)+\left(R_{l_{1}, k_{2}}^{c_{1}}-R_{l_{1}, k_{1}}^{c_{1}}\right)+R_{l_{1}, k_{1}}(t)}{\mathrm{c}}\right) d t .
\end{aligned}
$$

Similar to BiFBP the processing time of BiFFBP does not only depend on the number of aperture positions $N_{l}$ and the reconstructed SAR scene $\left(N_{x} \times N_{y}\right)$ but also the selections of subaperture $L$ and subimage $K$. However, the processing time of BiFFBP is also affected by the number of beamforming stages. As shown in [20] the processing time of FFBP in general and BiFFBP in particular is proportional to $\lambda N^{2} \log _{\lambda}(N)$, where $\lambda$ is a constant number of aperture positions in subapertures for all beamforming stages. In this case the processing time can be reduced with a factor of $(N / \lambda) / \log _{\lambda}(N)$ in comparison with that of BiGBP.

Equation (19) is available for BiFFBP where the values of the maximum dimension of the subimages $d_{k}$, the transmitter subaperture length $d_{t}$, and the receiver subaperture length $d_{r}$ are different from one beamforming stage to other beamforming stages. If the maximum dimension of the subimages decreases with a constant factor and the subaperture lengths increase with the same factor after every beamforming stage, the phase error will not be changed.

\section{RESULTS AND EVALUATIONS}

In this section we present some simulation results of the introduced algorithms and evaluations of their performance. Table I shows the system parameters of CARABAS-II [2] and the motion parameters of LORA [3] which are used in the simulations.

$\mathrm{BiFBP}$ is selected to test with different bistatic SAR geometries or configurations. The considered bistatic configurations are quasi-monostatic, i.e., 
transmitter and receiver mounted on a single platform, azimuth-invariant, i.e., transmitter and receiver mounted on two different platforms whose flight tracks are parallel, and general bistatic, i.e., transmitter and receiver mounted on two different platforms whose flight tracks are arbitrary. In the simulations the transmitter is simulated according to CARABAS-II's parameters, while the motion parameters of LORA also given in Table I are used to simulate the platform carrying the passive receiver.

The evaluation of the algorithm performance is based on a comparison among BiGBP, BiFBP, and BiFFBP in which BiGBP is the reference for the comparison. For comparison purposes only the general bistatic configuration needs to be taken into account.

\section{A. Testing with Different Bistatic Configurations}

In the following tests with three bistatic configurations, i.e., quasi-monostatic, azimuth-invariant bistatic, and general bistatic, the ground scene $1024 \mathrm{~m} \times 1024 \mathrm{~m}$ illuminated by these systems is simulated by a number of point-like scatterers. They are equally spaced and their radar cross section (RCS) is $\sigma=1 \mathrm{~m}^{2}$. The thermal noise and the clutter are excluded in the simulations. This ground scene is used in all simulations.

1) Quasi-Monostatic: In the first configuration the transmitter and receiver are carried by one platform and separated by a distance of $3 \lambda / 2$. This bistatic configuration has been used in LORA for ground moving target indication (GMTI) purposes. However, in this simulation, the CARABAS-II's parameters given in Table I are used to simulate such a bistatic SAR system. For this specific bistatic configuration, the traveling distance of a radar pulse can be approximated by the two-way traveling distance of that radar pulse with respect to the center of the transmitter and receiver. The SAR processing can therefore be relied on the monostatic SAR algorithms as shown in [32].

Also, for this special bistatic configuration, the maximum bistatic backprojection angle can be approximated to zero degree $\left(\alpha \approx 0^{\circ}\right.$ or $\left.\cos \alpha=1\right)$, the transmitter and receiver subaperture lengths are almost indentical $\left(d_{t} \approx d_{r}=d_{l}\right)$, and there is almost no difference between the minimum ranges $\left(R_{t, 0} \approx R_{r, 0}=\right.$ $R_{0}$ ). Equation (19) is simplified to

$$
\Delta \phi_{\max } \approx \frac{\pi}{2 \lambda_{c}} \frac{d_{k} d_{l}}{R_{0}} .
$$

The number of subapertures is arbitrarily selected by $L=320$, or in other words the subaperture length is given by $64 \times 0.9375 \mathrm{~m}$, while the selection of the subimage area is $64 \mathrm{~m} \times 64 \mathrm{~m}$ with the image sample of $0.5 \mathrm{~m} \times 0.5 \mathrm{~m}$. The number of subimages is shown to be $K=256$. According to (29) these selections assure that the phase error caused by BiFBP is about

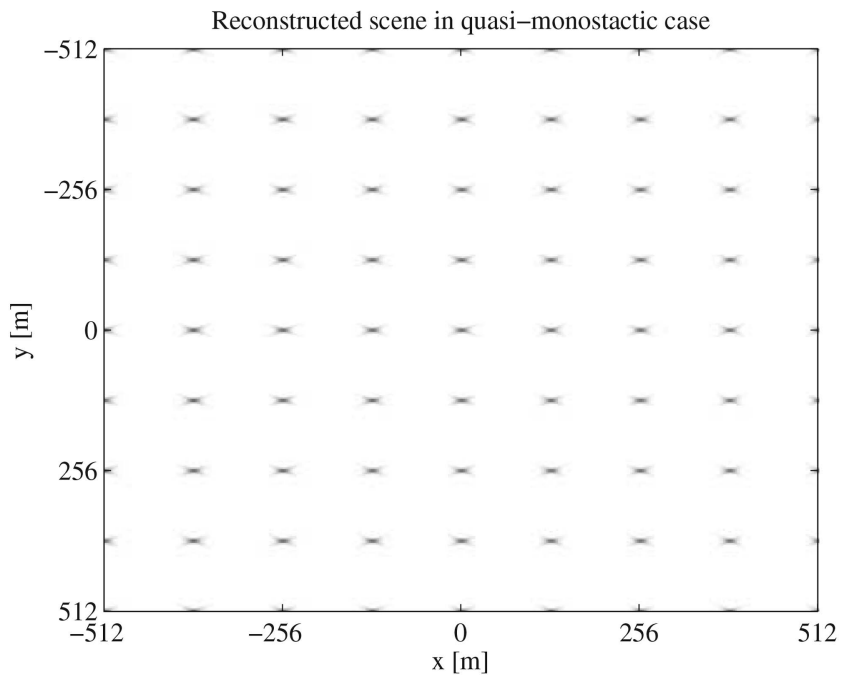

Fig. 7. Reconstructed SAR scene in quasi-monostatic case. BiFBP works with $L=47$ and $K=256$.

$\pi / 12$, which meets the demand of far field condition $\left(\Delta \phi_{\max } \leq \pi / 8\right)$.

Figure 7 shows the reconstructed SAR scene in the quasi-monostatic case. All scatterers in the reconstructed scene are well focused and appear in that SAR image as point targets. This SAR image is supposed to be more or less the same as a SAR image generated by a monostatic SAR system operating in the same frequency range. The general features of the point targets, i.e., point-like scattering, orthogonal and nonorthogonal sidelobes, are similar to those of the monostatic cases.

2) Azimuth-Invariant: For the azimuth-invariant configuration, the transmitter and receiver are carried by two different platforms. However, their flight tracks are parallel. This bistatic SAR configuration has been used to build a bistatic SAR system with LORA and SETHI [20]. Hence, in the following simulation, a transmitter is assumed to be carried by CARABAS-II while a passive receiver operates in the same frequency range by another platform whose motion parameters are similar to those of LORA.

As shown in Table I, there is also a small difference between the speeds of the two platforms. The space between the platforms is $2000 \mathrm{~m}$ in the $y$-direction and $800 \mathrm{~m}$ in $z$-direction. Hence, the maximum bistatic backprojection angle can still be approximated to zero degree in this case $\left(\alpha \approx 0^{\circ}\right)$.

In SAR scene reconstruction using BiFBP, the subaperture length and subimage area are also selected by $64 \times 0.9375 \mathrm{~m}$ and $64 \mathrm{~m} \times 64 \mathrm{~m}$ with the image sample of $0.5 \mathrm{~m} \times 0.5 \mathrm{~m}$, respectively. The phase error caused by BiFBP with these selections can therefore show to be still smaller than $\pi / 8$.

The reconstructed scene is given in Fig. 8 and is similar to the one shown in Fig. 7. The general features of the point targets in Fig. 7 can also be observed in Fig. 8. 


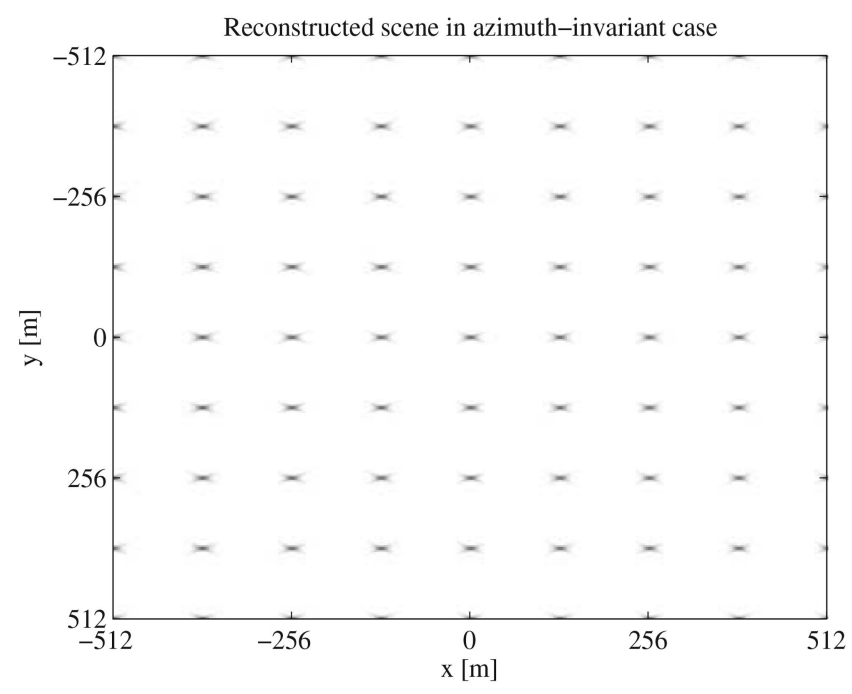

Fig. 8. Reconstructed SAR scene in azimuth-invariant case. BiFBP works with $L=47$ and $K=256$.

3) General: The general bistatic configuration is interpreted as a bistatic configuration without limitations of platforms' motion parameters. In other words the platforms can fly at any altitude, with any speed, and in any direction. In the following simulation the simulated bistatic SAR system is built on monostatic systems CARABAS-II and another platform with the same LORA motion parameters. The transmitter of CARABAS-II is utilized in this bistatic system while a passive receiver is assumed to be carried by the other platform. The flight tracks form an arbitrary angle of $60^{\circ}$. This bistatic SAR system can be considered as a UWB low-frequency bistatic SAR system since the system operates in the VHF-band, the fractional bandwidth of the radar signal is about 1.16 , and the integration angle with respect to the transmitter platform is up to $130^{\circ}$.

In [31] the phase error calculation based on (19) for this bistatic SAR system is presented. The limits of subaperture length and subimage area to keep the phase error caused by BiFBP below $\pi / 8$ are $32 \times 0.9375 \mathrm{~m}$ and $64 \mathrm{~m} \times 64 \mathrm{~m}$, respectively. These values correspond to $L=640$ and $K=256$ and are used for BiFBP in the SAR scene reconstruction.

Figure 9 shows the reconstructed SAR scene with the simulated data of this SAR system. We suppose that the point-like scatterers on the ground scene appear as point targets in the SAR image since the general features of a point-like scatterer illuminated by a UWB SAR system such as point-like scattering and orthogonal and nonorthogonal sidelobes can be observed. However, the point targets are inclined with a certain angle. This inclination is believed to be dependent on the motion parameters of the platforms as for the quasi-monostatic and azimuth-invariant configurations, there is almost no inclination.

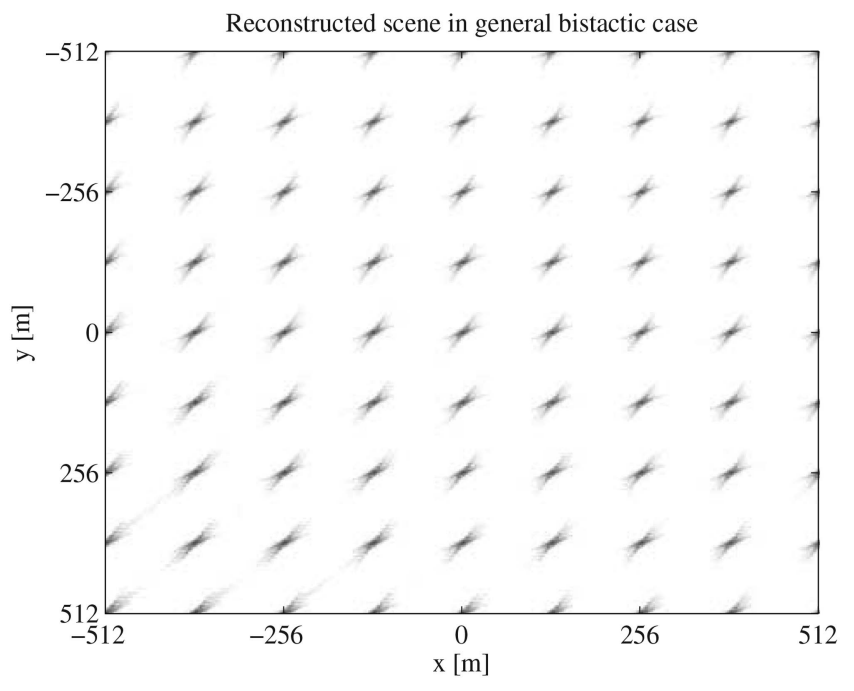

Fig. 9. Reconstructed SAR scene in general bistactic case. BiFBP works with $L=640$ and $K=256$.

The simulation results with three different bistatic SAR configurations presented here indicate that BiFBP is not limited by any bistatic configuration. This is also true for BiFFBP as shown in the following evaluation of BiFBP and BiFFBP performance.

To evaluate the performance of the bistatic algorithms, we can compare SAR images of a point-like scatterer on the ground scene reconstructed with BiGBP, BiFBP, and BiFFBP. The SAR image processed with BiGBP should be the reference for this comparison since this algorithm does not cause any phase error in theory. In the following comparison the bistatic SAR system with the general bistatic configuration is considered. On account of the local processing characteristic of the time-domain algorithms, we can reconstruct small SAR images $128 \mathrm{~m} \times 128 \mathrm{~m}$ showing the point-like scatterer located at the center of the ground scene. This position associates with the maximum errors possibly caused by algorithms.

B. Evaluating the Performance of the Bistatic
Algorithms

In SAR scene reconstruction BiFBP works with the same subaperture length as in the previous simulation $32 \times 0.9375 \mathrm{~m}$. For illustration and comparison purposes, the image sample is reduced $0.125 \mathrm{~m} \times 0.125 \mathrm{~m}$. The number of SAR image samples is $1024 \times 1024$ samples. With the subimage area of $64 \mathrm{~m} \times 64 \mathrm{~m}$, the number of subimages is $K=4$. For BiFFBP with two beamforming stages, we select $L_{1}=640$ and $K_{1}=4$ for the the first beamforming stage. These values are identical to those of BiFBP. A factor of two (2) is applied for the next beamforming stage, i.e., $L_{2}=320$ and $K_{2}=16$. The second-order beams are then used to form SAR images. 


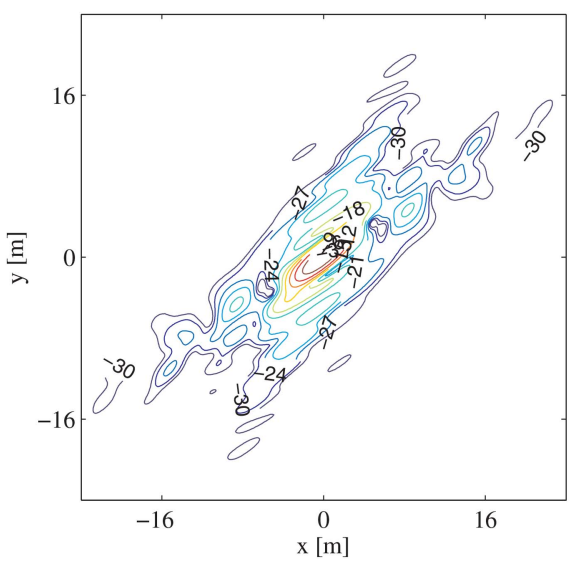

(a)

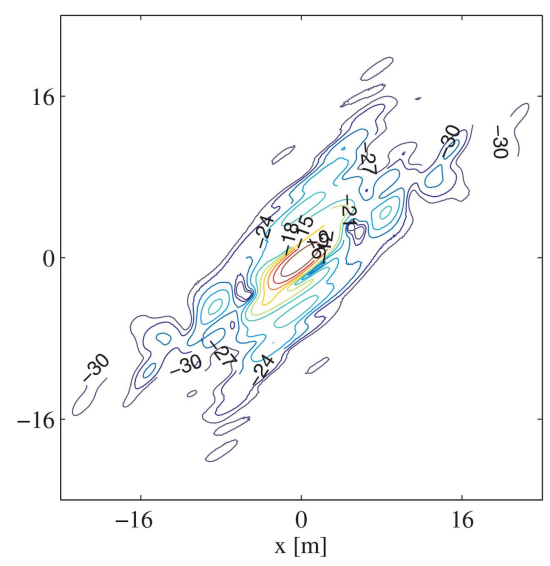

(b)

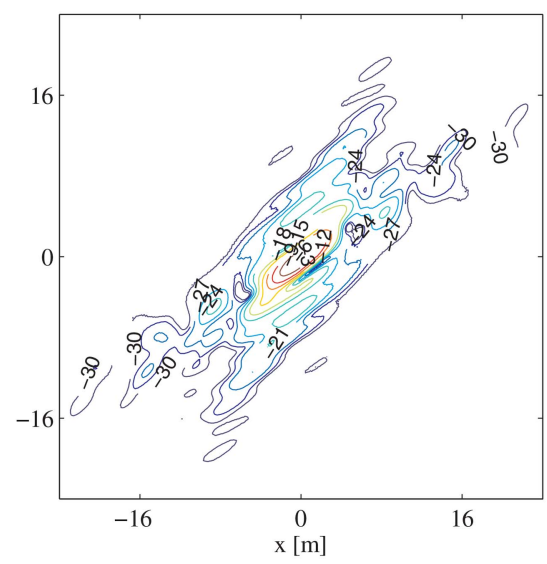

(c)

Fig. 10. Part of SAR images of point-like scatterer located at center of ground scene reconstructed with time-domain algorithms and represented in contour plots. (a) BiGBP. (b) BiFBP. (c) BiFFBP.

Figures 10(a), (b), and (c) show a part of the SAR images of the point-like scatterer located at the center of the ground scene reconstructed with BiGBP, $\mathrm{BiFBP}$, and BiFFBP and represented in contour plots. A contour level from $-30 \mathrm{~dB}$ to $0 \mathrm{~dB}$ and a contour step of $-3 \mathrm{~dB}$ are applied in these plots.

Since there is no definition of SAR image quality assessment for bistatic cases, the comparison of the algorithms' performance can only rely on observations. At first look the SAR images of the point-like scatterer built with $\mathrm{BiGBP}, \mathrm{BiFBP}$, and BiFFBP are almost identical. There seems to be no loss in spatial resolutions due to phase errors caused by BiFBP and BiFFBP. The sidelobe levels generated by BiFBP are a little higher than the ones by BiGBP and a little lower than the ones by BiFFBP. If there exists an integrated sidelobe ratio (ISLR) measurement for bistatic SAR cases, this value should be minimum for BiGBP and maximum for BiFFBP. Similarly, the effects of phase error may not be large enough to significantly change the peak sidelobe ratio (PSLR) measurement results if there are any.

To measure the real processing times, the algorithms BiGBP, BiFBP, and BiFFBP have been implemented in Matlab version 7.3.0 and on a computer with a $2.94 \mathrm{GHz}$ Intel processor and $4 \mathrm{~GB}$ RAM. Under our implementations the processing times are about $11707 \mathrm{~s}$ for BiGBP, $529 \mathrm{~s}$ for BiFBP, and $407 \mathrm{~s}$ for BiFFBP. Comparing the real processing time of BiFBP and BiFFBP with BiGBP, the reduction factors in processing time are about 22 and 29 times, respectively. Although the factors are significant they are still smaller than the optimum factors which can be obtained with the fast time-domain algorithms $(\sqrt{N}$ for BiFBP and $(N / \lambda) / \log _{\lambda}(N)$ for BiFFBP). This can be partially explained by the implementations of the algorithms. Also, the parameter selections for the algorithms are not optimum due to the far-field condition. However, the measured results clearly give us a hint of the processing time reduction.

\section{CONCLUSION}

In this study the ability to apply GBP directly to bistatic SAR is presented. Then we introduce two fast time-domain algorithms to process bistatic SAR in general and UWB bistatic SAR in particular. A subaperture and subimage basis is utilized in these algorithms. The processing is divided into two main stages: beamforming and local backprojection. The difference between BiFBP and BiFFBP is only the number of beamforming substages. The introduced algorithms are tested with different SAR configurations and then their performance is compared with that of BiGBP. The testing results show that BiFBP and BiFFBP inherit the time-domain characteristics and are not limited by any bistatic SAR configuration.

\section{ACKNOWLEDGMENT}

The authors would like to thank the Swedish Defence Research Agency, Saab Bofors Dynamics, Saab Electronic Defence Systems, and RUAG Space for their cooperation.

\section{REFERENCES}

[1] Federal Communications Commission (FCC) Revision of part 15 of the commission's rules regarding ultra-wideband systems: First report and order. Federal Communications Commission (FCC), FCC 02-48, Apr. 2002, p. 36.

[2] Gustavsson, A., et al.

Development and operation of an airborne VHF SAR system-lessons learned.

Proceedings of the IEEE International Geoscience and Remote Sensing Symposium (IGARSS'98), vol. 1, Seattle, WA, July 1998, pp. 458-462.

[3] Ulander, L. M. H. and Hellsten, H.

Low-frequency ultrawideband array-antenna SAR for stationary and moving target imaging.

Proceedings of SPIE, vol. 3704, Radar Sensor Technology IV, Orlando, FL, Apr. 1999, pp. 149-158, DOI: $10.1117 / 12.354592$. 
[4] Vu, V. T., Sjögren, T. K., and Pettersson, M. I.

On synthetic aperture radar azimuth and range resolution equations.

IEEE Transactions on Aerospace and Electronic Systems, 48, 2 (2012), 1764-1769.

[5] Ulander, L. M. H., et al.

Change detection for low-frequency SAR ground surveillance.

IEE Proceedings—Radar, Sonar and Navigation, 152, 6 (2005), 413-420.

[6] Vu, V. T., et al.

Moving targets detection by focusing in UWB

SAR-Theory and experimental results.

IEEE Transactions on Geoscience and Remote Sensing, 48, 10 (2010), 3799-3815.

[7] Sjögren, T. K., et al.

A method for moving target speed estimation and refocusing in SAR.

IEEE Transactions on Aerospace and Electronic Systems, 48, 3 (2012), 2426-2436.

[8] Goodman, R., Tummala, S., and Carrara, W.

Issues in ultra-wideband, widebeam SAR image

formation.

Proceedings of the IEEE International Radar Conference, Alexandria, VA, May 1995, pp. 479-485.

[9] Cummings, J. G. and Bennett, J. R.

Digital processing of Sesat SAR data.

Proceedings of the International Conference on Acoustics, Speech and Signal Processing, Washington, D.C., Apr. 1979, pp. 110-118.

[10] Cafforio, C., Prati, C., and Rocca, F

SAR data focusing using seismic migration techniques. IEEE Transactions on Aerospace and Electronic Systems, 27 (Mar. 1991), 194-207.

[11] Raney, R. K., et al.

Precision SAR processing using chirp scaling.

IEEE Transactions on Geoscience and Remote Sensing, 32, 4 (1994), 786-799.

[12] Vu, V. T., et al.

An impulse response function for evaluation of

ultrawideband SAR imaging.

IEEE Transactions on Signal Processing, 58, 7 (2010), 3927-3932.

[13] Wang, R. Y., et al.

Focus FMCW SAR data using wavenumber domain algorithm.

IEEE Transactions on Geoscience and Remote Sensing, 48, 4 (2010), 2109-2118.

[14] Vu, V. T., Sjögren, T. K., and Pettersson, M. I.

A comparison between fast factorized backprojection and frequency-domain algorithms in UWB low frequency SAR.

Proceedings of the IEEE International Geoscience and Remote Sensing Symposium (IGARSS 2008), Boston, MA, July 2008, pp. 1293-1296.

[15] Andersson, L. E.

On the determination of a function from spherical averages.

SIAM Journal on Mathematical Analysis, 19, 1 (1988), 214-232.

[16] Walterscheid, I., et al.

Bistatic image processing for a hybrid SAR experiment between TerraSAR-X and PAMIR.

Proceedings of the IEEE International Geoscience and Remote Sensing Symposium (IGARSS 2006), Denver, CO, Aug. 2006, pp. 1934-1937.

[17] Brenner, A. R. and Ender, J. H. G.

Demonstration of advanced reconnaissance techniques with the airborne SAR/GMTI sensor PAMIR.

IEE Proceedings-Radar, Sonar and Navigation, 153, 2

(2006), 152-162.
[18] Roth, A. and Werninghaus, R.

Status of the TerraSAR-X mission.

Proceedings of the IEEE International Geoscience and Remote Sensing Symposium (IGARSS 2005), Seoul, Korea, July 2005, pp. 4868-4869.

[19] Baque, R., et al. LORAMbis-A bistatic VHF/UHF SAR experiment for FOPEN.

Proceedings of the 2010 IEEE Radar Conference, Washington, D.C., May 2010, pp. 832-837.

[20] Ulander, L. M. H., et al.

Fast factorized back-projection for bistatic SAR processing.

Proceedings of the 2010 8th European Conference on Synthetic Aperture Radar (EUSAR 2010), Aachen, Germany, June 2010, pp. 1-4.

[21] Neo, Y. L., Wong, F. H., and Cumming, I. G. Processing of azimuth-invariant bistatic SAR data using the range Doppler algorithm.

IEEE Transactions on Geoscience and Remote Sensing, 46, 1 (2008), 14-21.

[22] Walterscheid, I., et al.

Bistatic SAR processing using an omega-k type algorithm.

Proceedings of the IEEE International Geoscience and Remote Sensing Symposium (IGARSS 2005), vol. 2, Seoul, Korea, July 2005, pp. 1064-1067.

[23] Wong, F. H., Cumming, I. G., and Neo, Y. L.

Focusing bistatic SAR data using the nonlinear chirp scaling algorithm.

IEEE Transactions on Geoscience and Remote Sensing, 46, 9 (2008), 2493-2505.

[24] Wang, R. Y., et al.

Processing the azimuth-variant bistatic SAR data by using monostatic imaging algorithm based on $2 \mathrm{D}$ principle of stationary phase.

IEEE Transactions on Geoscience and Remote Sensing, 49, 10 (2011), 3504-3520.

[25] Vu, V. T., Sjögren, T. K., and Pettersson, M. I.

Fast backprojection algorithm for UWB bistatic SAR. Proceedings of the 2011 IEEE Radar Conference (Radar 2011), Kansas City, MO, May 2011, pp. 431-434.

[26] Jakowatz, C. V., Wahl, D. E., and Yocky, D. A. A beamforming algorithm for bistatic SAR image formation.

Proceedings of SPIE, vol. 7699, Algorithms for Synthetic Aperture Radar Imagery XVII, Orlando, FL, Apr. 2010, pp. 769902-1-769902-6.

[27] Rodriguez-Cassola, M., et al.

Efficient time-domain focussing for general bistatic SAR configurations: Bistatic fast factorised backprojection. Proceedings of the 2010 8th European Conference on Synthetic Aperture Radar (EUSAR 2010), Aachen, Germany, June 2010, pp. 1050-1053.

[28] Vu, V. T., Sjögren, T. K., and Pettersson, M. I. SAR imaging in ground plane using fast backprojection for mono- and bistatic cases.

Proceedings of the 2010 IEEE Radar Conference (RADAR 2012), Atlanta, GA, May 2012, pp. 184-189.

[29] Vu, V. T., Sjögren, T. K., and Pettersson, M. I.

Fast factorized backprojection algorithm for UWB bistatic SAR image reconstruction.

Proceedings of the IEEE International Geoscience and Remote Sensing Symposium (IGARSS 2011), Vancouver, Canada, July 2011, pp. 4237-4240. 

May 1998, pp. 489-492.

[31] Vu, V. T., Sjögren, T. K., and Pettersson, M. I.

Phase error calculation for time-domain bistatic SAR algorithms.

IEEE Transactions on Aerospace and Electronic Systems, 49, 1 (Jan. 2013), 38-46.
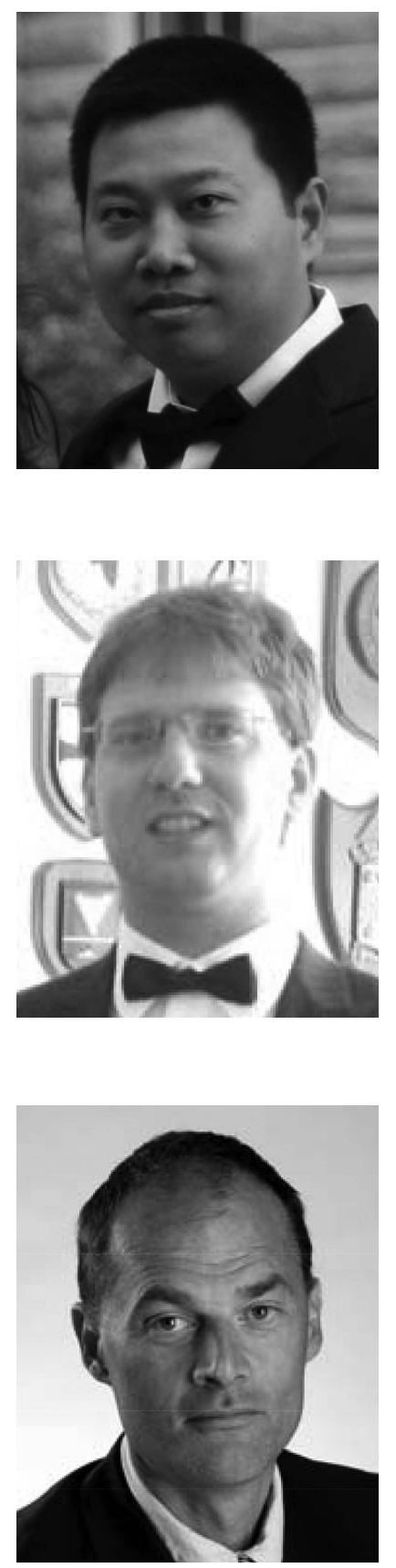

Viet Thuy Vu (SM'06-M'12) was born in Hanoi, Vietnam. He received his Diploma degree in electronics at the Hanoi University of Technology, Vietnam, his Master of Science degree in communication engineering at the University of Duisburg-Essen, Germany, the Licentiate in applied signal processing at the Blekinge Institute of Technology (BTH), Sweden, and his Doctoral degree in applied signal processing also at BTH in 2011.

$\mathrm{He}$ is currently a postdoctoral research associate at BTH. His research interests are mono- and bi-static UWB SAR signal processing, applications of UWB SAR in moving target detection, and estimation.

$\mathrm{Dr}$. $\mathrm{Vu}$ is the author and coauthor of about 40 professional publications. In 2007 he was awarded the Student Paper Prize at the IET International Conference on Radar Systems.

Thomas K. Sjögren (SM'06-M'13) was born in Sundsvall, Sweden in 1982. He received his M.Sc. in space engineering from Luleå University of Technology (LTU), Sweden in 2006. In 2010 he received his Licentiate degree and in 2012 his Ph.D. degree in applied signal processing at Blekinge Institute of Technology (BTH), Sweden.

Dr. Sjögren's main research interests include detection of moving targets and estimation of target parameters in SAR, especially wideband SAR.

Mats I. Pettersson received his M.Sc. in engineering physics in 1993, Licentiate degree in radio and space science in 1995, and his Ph.D. in signal processing in 2000, all from Chalmers University of Technology, Gothenburg, Sweden.

He worked at Ericsson Mobile Communication as a patent project manager and for almost ten years he was employed at the Swedish Defence Research Agency (FOI). At FOI he worked as a research manager and a researcher, mainly focusing on ultrawideband low frequency SAR systems. Since 2005 he has been employed at the Blekinge Institute of Technology where he is a professor in electrical engineering and the research director in the Department of Electrical Engineering. His work is related to radar and sonar signal processing where his main interests are SAR processing, space time adaptive processing (STAP), and sparse passive underwater arrays. 